\title{
Coherent and incoherent processes and the LPM effect in oriented single crystals at high-energy
}

\author{
V. N. Baier*, V. M. Katkov \\ Budker Institute of Nuclear Physics, \\ Novosibirsk, 630090, Russia
}

\begin{abstract}
The process of radiation from high-energy electron and electron-positron pair production by a photon in oriented single crystal is considered using the method which permits inseparable consideration of both coherent and incoherent mechanisms of photon emission from an electron and of pair creation by a photon and includes the action of field of axis (or plane) as well as the multiple scattering of radiating electron or particles of the created pair (the Landau-Pomeranchuk-Migdal (LPM) effect). The total intensity of radiation and total probability pair creation are calculated. The theory, where the energy loss of projectile has to be taken into account, and found probabilities of pair creation agree quite satisfactory with available CERN data. From obtained results it follows that multiple scattering appears only for relatively low energy of radiating electron or a photon, while at higher energies the field action excludes the LPM effect.
\end{abstract}

\section{INTRODUCTION}

The mechanisms of basic electromagnetic process in oriented single crystal (radiation and pair production by a photon) differ substantially from the mechanisms of independent photon emission from electron or pair photoproduction at separate centers acting in an amorphous medium (the Bethe-Heitler mechanisms). In crystal the coherent interaction of an electron (or a photon) with many centers occurs. Under some generic assumptions the general theory of the coherent radiation mechanism was developed in ${ }^{1}$ (of the coherent pair creation mechanism in ${ }^{2}$ ). Recently authors developed the new approach to analysis of pair creation by a photon ${ }^{3}$ and radiation ${ }^{4}$ in oriented crystals. This approach not only permits indivisible consideration of both the coherent and incoherent mechanisms of radiation (or pair creation by a photon) but also gives insight on the LandauPomeranchuk-Migdal (LPM) effect (influence of multiple scattering) on the considered processes.

The properties of process are connected directly with details of motion of emitting particle (or particles of created pair). The momentum transfer from a particle to a crystal we present in a form $\mathbf{q}=\langle\mathbf{q}\rangle+\mathbf{q}_{s}$, where $\langle\mathbf{q}\rangle$ is the mean value of momentum transfer calculated with averaging over thermal(zero) vibrations of atoms in a crystal. The motion of particle in an averaged potential of crystal, which corresponds to the momentum transfer $\langle\mathbf{q}\rangle$, determines the coherent mechanism of process. The term $\mathbf{q}_{s}$ is attributed to the random collisions of particle which define the incoherent radiation (pair creation). Such random collisions we will call "scattering" since $\left\langle\mathbf{q}_{s}\right\rangle=0$. If the formation length of the process is large with respect to distances between atoms forming the axis, the additional averaging over the atom position should be performed.

If the electron (or photon) angle of incidence $\vartheta_{0}$ (the angle between the electron momentum $\mathbf{p}$ (or photon momentum $\mathbf{k}$ ) and the axis (or plane)) is small $\vartheta_{0} \ll V_{0} / m$, where $V_{0}$ is the characteristic scale of the potential, the field $E$ of the axis (or plane) can be considered constant over the process formation length and the constant-field approximation is valid. In this case the behavior of radiation probability is determined by the parameter $\chi$ and the pair production probability is determined by the parameter $\kappa$ :

$$
\chi=\frac{\varepsilon}{m} \frac{E}{E_{0}}, \quad \kappa=\frac{\omega}{m} \frac{E}{E_{0}},
$$

where $\varepsilon(\omega)$ is the electron (photon) energy, $m$ is the electron mass, $E_{0}=m^{2} / e=1.32 \cdot 10^{16} \mathrm{~V} / \mathrm{cm}$ is the critical field, the system $\hbar=c=1$ is used.

*V.N.Baier@inp.nsk.su 
The very important feature of coherent radiation (or coherent pair creation) mechanism is the strong enhancement of its probability at high energies (from factor $\sim 10$ for main axes in crystals of heavy elements like tungsten to factor $\sim 160$ for diamond) comparing with the Bethe-Heitler mechanism which takes place in an amorphous medium. If $\vartheta_{0} \gg V_{0} / m$ the theory passes over to the coherent bremsstrahlung theory or the coherent pair production theory $\left(\mathrm{se}^{6-8}\right)$. Side by side with coherent mechanism the incoherent mechanism of radiation is acting. In oriented crystal this mechanism changes also with respect to an amorphous medium. ${ }^{9}$ The details of theory and description of experimental study of radiation and pair creation which confirms the mentioned enhancement can be found in. ${ }^{8}$ The study of radiation and pair creation in oriented crystals is continuing and new experiments are performed recently. ${ }^{10-13}$

At high energies the multiple scattering of radiating electron or particles of created pair (the LPM effect) suppresses radiation (or pair creation) probability when $\varepsilon \geq \varepsilon_{e}$ (or $\omega \geq \omega_{e}$ ). In an amorphous medium (or in crystal in the case of random orientation) the characteristic electron energy starting from which the LPM effect becomes essential is $\varepsilon_{e} \sim 2.5 \mathrm{TeV}$ for heavy elements ${ }^{14}$ and this value is inversely proportional to the density. In the vicinity of crystalline axis (just this region gives the crucial contribution to the Bethe-Heitler mechanism) the local density of atoms is much higher than average one and for heavy elements and at low temperature the gain could attain factor $\sim 10^{3}$. So in this situation the characteristic electron energy can be $\varepsilon_{0} \sim 2.5 \mathrm{GeV}$ and this energy is significantly larger than "threshold" energy $\varepsilon_{t}$ starting from which the probability of coherent radiation exceeds the incoherent one. For pair photoproduction the characteristic photon energies are 4 times larger: $\omega_{e}=4 \varepsilon_{e} \sim 10 \mathrm{TeV}$ for heavy elements in an amorphous medium and in crystal $\omega_{0}=\omega_{e} / \xi(0) \sim 10 \mathrm{GeV}$. The last energy is of the order of the threshold energy $\omega_{t}$ for which the probability of pair creation in the axis field becomes equal to the BetheMaximon probability, see Sec.12.2 and Table 12.1 in. ${ }^{8}$ It should be noted that the main contribution into the multiple scattering gives the small distance from axis where the field of crystalline axis attains the maximal value. For the same reason the LPM effect in oriented crystals originates in the presence of crystal field and nonseparable from it. This means that in problem under consideration we have both the dense matter with strong multiple scattering and high field of crystalline axis.

Below we consider case $\vartheta_{0} \ll V_{0} / \mathrm{m}$. Than the distance of an electron from axis $\rho$ as well as the transverse field of the axis can be considered as constant over the formation length. For an axial orientation of crystal the ratio of the atom density $n(\rho)$ in the vicinity of an axis to the mean atom density $n_{a}$ is

$$
\frac{n(x)}{n_{a}}=\xi(x)=\frac{x_{0}}{\eta_{1}} e^{-x / \eta_{1}}, \quad \varepsilon_{0}=\frac{\varepsilon_{e}}{\xi(0)},
$$

where

$$
x_{0}=\frac{1}{\pi d n_{a} a_{s}^{2}}, \quad \eta_{1}=\frac{2 u_{1}^{2}}{a_{s}^{2}}, \quad x=\frac{\rho^{2}}{a_{s}^{2}},
$$

Here $\rho$ is the distance from axis, $u_{1}$ is the amplitude of thermal vibration, $d$ is the mean distance between atoms forming the axis, $a_{s}$ is the effective screening radius of the axis potential (see Eq. $(9.13)$ in $^{8}$ )

$$
U(x)=V_{0}\left[\ln \left(1+\frac{1}{x+\eta}\right)-\ln \left(1+\frac{1}{x_{0}+\eta}\right)\right] .
$$

The local value of parameters $\chi(x)(\kappa(x))$, see Eq.(1), which determines the radiation (pair creation) probability in the field Eq.(4) is

$$
\chi(x)=-\frac{d U(\rho)}{d \rho} \frac{\varepsilon}{m^{3}}=\chi_{s} f_{a}, \quad f_{a}=\frac{2 \sqrt{x}}{(x+\eta)(x+\eta+1)}, \quad \chi_{s}=\frac{V_{0} \varepsilon}{m^{3} a_{s}} \equiv \frac{\varepsilon}{\varepsilon_{s}}, \quad \kappa(x)=\kappa_{s} f_{a}, \quad \kappa_{s}=\frac{V_{0} \omega}{m^{3} a_{s}} \equiv \frac{\omega}{\omega_{s}} .
$$

The parameters of the axial potential for the ordinarily used crystals are given in Table $9.1 \mathrm{in}^{8}$ The particular calculation below will be done for tungsten and germanium crystals studied in. ${ }^{10,12}$ The relevant parameters are given in Table 1. 
TABLE 1 Parameters of radiation (pair creation) process in the tungsten (the axis $<111>$ ) and germanium (the axis $<110>$ ) crystals for different temperatures T, the energies $\varepsilon$ and $\omega$ are in $\mathrm{GeV}$

\begin{tabular}{|c|c|c|c|c|c|c|c|c|c|c|c|}
\hline Crystal & $\mathrm{T}(\mathrm{K})$ & $V_{0}(\mathrm{eV})$ & $x_{0}$ & $\eta_{1}$ & $\eta$ & $\varepsilon_{0}$ & $\varepsilon_{t}$ & $\varepsilon_{s}\left(\omega_{s}\right)$ & $\varepsilon_{m}\left(\omega_{m}\right)$ & $\omega_{0}$ & $h$ \\
\hline $\mathrm{W}$ & 293 & 413 & 39.7 & 0.108 & 0.115 & 7.43 & 0.76 & 34.8 & 14.35 & 29.7 & 0.348 \\
\hline $\mathrm{W}$ & 100 & 355 & 35.7 & 0.0401 & 0.0313 & 3.06 & 0.35 & 43.1 & 8.10 & 12.25 & 0.612 \\
\hline $\mathrm{Ge}$ & 100 & 114.5 & 19.8 & 0.064 & 0.0633 & 59 & 0.85 & 179 & 51 & 236 & 0.459 \\
\hline
\end{tabular}

\section{PROCESSES IN LIMITING CASES}

\subsection{Radiation}

It is useful to compare the characteristic energy $\varepsilon_{0}$ (or $\omega_{0}$ for pair creation) with "threshold" energy $\varepsilon_{t}$ (or $\omega_{t}$ for pair creation) for which the radiation intensity (pair creation probability) in the axis field becomes equal to the Bethe-Maximon one. Since the maximal value of parameter $\chi(x)$ :

$$
\chi_{m}=\chi\left(x_{m}\right), \quad \kappa_{m}=\kappa\left(x_{m}\right), \quad x_{m}=\frac{1}{6}(\sqrt{1+16 \eta(1+\eta)}-1-2 \eta), \quad \chi_{m}=\frac{\varepsilon}{\varepsilon_{m}}, \quad \kappa_{m}=\frac{\omega}{\omega_{m}}
$$

is small for such electron energy $\left(\varepsilon_{t} \ll \varepsilon_{m}\right)$, one can use the decomposition of radiation intensity over powers of $\chi$ (see Eq.(4.52) $\left.i^{8}\right)$ and carry out averaging over $x$. Retaining three terms of decomposition we get

$$
\begin{aligned}
& I^{F}=\frac{8 \alpha m^{2} \chi_{s}^{2}}{3 x_{0}}\left(a_{0}(\eta)-a_{1}(\eta) \chi_{s}+a_{2}(\eta) \chi_{s}^{2}+\ldots\right) \\
& a_{0}(\eta)=(1+2 \eta) \ln \frac{1+\eta}{\eta}-2, \quad a_{1}(\eta)=\frac{165 \sqrt{3} \pi}{64}\left[\frac{1}{\sqrt{\eta}}-\frac{1}{\sqrt{1+\eta}}-4(\sqrt{1+\eta}-\sqrt{\eta})^{3}\right], \\
& a_{2}(\eta)=64\left[(1+2 \eta)\left(\frac{1}{\eta(1+\eta)}+30\right)-12(1+5 \eta(1+\eta)) \ln \frac{1+\eta}{\eta}\right] .
\end{aligned}
$$

The intensity of incoherent radiation in low energy region $\varepsilon \leq \varepsilon_{t} \ll \varepsilon_{m}$ is (see Eq.(21.16) in ${ }^{8}$ and Eq.(41) below)

$$
I^{i n c}=\frac{\alpha m^{2}}{4 \pi} \frac{\varepsilon}{\varepsilon_{e}} g_{0 r}\left[1+34.4\left(\overline{\chi^{2} \ln \chi}+2.54 \overline{\chi^{2}}\right)\right], \quad g_{0 r}=1+\frac{1}{L_{0}}\left[\frac{1}{18}-h\left(\frac{u_{1}^{2}}{a^{2}}\right)\right], \quad \bar{f}=\int_{0}^{\infty} f(x) e^{-\frac{x}{\eta_{1}}} \frac{d x}{\eta_{1}},
$$

where

$$
\begin{aligned}
& \varepsilon_{e}=\frac{m}{16 \pi Z^{2} \alpha^{2} \lambda_{c}^{3} n_{a} L_{0}}, \quad L_{0}=\ln (m a)+\frac{1}{2}-f(Z \alpha), \quad h(z)=-\frac{1}{2}\left[1+(1+z) e^{z} \operatorname{Ei}(-z)\right], \quad a=\frac{111 Z^{-1 / 3}}{m}, \\
& f(\xi)=\operatorname{Re}[\psi(1+i \xi)-\psi(1)]=\sum_{n=1}^{\infty} \frac{\xi^{2}}{n\left(n^{2}+\xi^{2}\right)}
\end{aligned}
$$

here $\psi(z)$ is the logarithmic derivative of the gamma function, $\operatorname{Ei}(z)$ is the integral exponential function, $f(\xi)$ is the Coulomb correction. For $\chi=0$ this intensity differs from the Bethe-Maximon intensity only by the term $h\left(u_{1}^{2} / a^{2}\right)$ which reflects the nonhomogeneity of atom distribution in crystal. For $u_{1} \ll a$ one has $h\left(u_{1}^{2} / a^{2}\right) \simeq-(1+C) / 2+\ln \left(a / u_{1}\right), C=0.577$.. and so this term characterizes the new value of upper boundary of impact parameters $u_{1}$ contributing to the value $<\mathbf{q}_{s}^{2}>$ instead of screening radius $a$ in an amorphous medium.

Conserving in Eq.(7) only the main (the first) term of decomposition, which corresponds to the classical radiation intensity, neglecting the corrections in Eq.(8) $\left(g_{0}=1, \chi=0\right)$, using the estimate $V_{0} \simeq Z \alpha / d$ and Eqs.(3), (5), we get

$$
\varepsilon_{t} \simeq \frac{3 L_{0} d m^{2}}{2 \pi a_{0}(\eta)}=63 \frac{L_{0} d}{a_{0}(\eta)} \mathrm{MeV}
$$


where the distance $d$ is taken in units $10^{-8} \mathrm{~cm}$. Values of $\varepsilon_{t}$ found using this estimate for tungsten, axis $<111>, d=2.74 \cdot 10^{-8}$ $\mathrm{cm}$ are consistent with points of intersection of coherent and incoherent intensities in Fig.1 (see Table 1). For some usable crystals (axis $<111>$, room temperature) one has from Eq.(10)

$$
\varepsilon_{t}\left(\mathrm{C}_{(\mathrm{d})}\right) \simeq 0.47 \mathrm{GeV}, \quad \varepsilon_{t}(\mathrm{Si}) \simeq 2.0 \mathrm{GeV}, \quad \varepsilon_{t}(\mathrm{Ge}) \simeq 1.7 \mathrm{GeV}
$$

so this values of $\varepsilon_{t}$ are somewhat larger than in tungsten except the diamond very specific crystal where value of $\varepsilon_{t}$ is close to tungsten one.

For large values of the parameter $\chi_{m}\left(\varepsilon \gg \varepsilon_{m}\right)$ the incoherent radiation intensity is suppressed due to the action of the axis field. In this case the local intensity of radiation can by written as (see Eq.(7.129) in ${ }^{8}$ )

$$
I^{i n c}=\frac{29 \Gamma(1 / 3)}{3^{1 / 6} 2430} \frac{\varepsilon}{\varepsilon_{e}} \frac{\alpha m^{2}}{\chi^{2 / 3}(x)}\left[g_{0 r}+\frac{1}{L_{0}}\left(0.727+\frac{\ln \chi(x)}{3}\right)\right] .
$$

Here we have taken into account that

$$
\ln \frac{1}{\gamma \vartheta_{1}}=\ln (m a) \rightarrow \ln (m a)-h\left(\frac{u_{1}^{2}}{a^{2}}\right)-f(Z \alpha)=L_{0}-h\left(\frac{u_{1}^{2}}{a^{2}}\right)-\frac{1}{2} .
$$

Averaging the function $(\chi(x))^{-2 / 3}$ and $\ln \chi(x)(\chi(x))^{-2 / 3}$ over $x$ according with Eq.(8) one can find the effective value of upper boundary of the transverse momentum transfer $\left(\propto m \chi_{m}^{1 / 3}\right.$ instead of $\left.m\right)$ which contributes to the value $<\mathbf{q}_{s}^{2}>$. Using the obtained results we determine the effective $\log$ arithm $L$ by means of interpolation procedure

$$
L=L_{0} g_{r}, \quad g_{r}=g_{0 r}+\frac{1}{6 L_{0}} \ln \left(1+70 \chi_{m}^{2}\right) .
$$

Let us introduce the local characteristic energy of electron (see Eq.(2))

$$
\varepsilon_{c}(x)=\frac{\varepsilon_{e}\left(n_{a}\right)}{\xi(x) g_{r}}=\frac{\varepsilon_{0}}{g_{r}} e^{x / \eta_{1}}
$$

In this notations the contribution of multiple scattering into the local intensity for small values of $\chi_{m}$ and $\varepsilon / \varepsilon_{0}$ has a form (see Eq.(15) in ${ }^{15}$ and Eq.(50) below)

$$
I^{L P M}(x)=-\frac{\alpha m^{2}}{4 \pi} \frac{\varepsilon}{\varepsilon_{c}(x)}\left[\frac{4 \pi \varepsilon}{15 \varepsilon_{c}(x)}\left(1+\frac{171 \sqrt{3}}{16} \chi(x)\right)+\frac{64 \varepsilon^{2}}{21 \varepsilon_{c}^{2}(x)}\left(\ln \frac{\varepsilon}{\varepsilon_{c}(x)}+2.04\right)\right] .
$$

Integrating this expression over $x$ with the weight $1 / x_{0}$ we get

$$
I^{L P M}=\frac{\alpha m^{2}}{4 \pi} \frac{\varepsilon}{\varepsilon_{e}} g_{r}\left[-\frac{2 \pi \varepsilon g_{r}}{15 \varepsilon_{0}}(1+37 \mu)+\frac{64}{63} \frac{\varepsilon^{2} g_{r}^{2}}{\varepsilon_{0}^{2}}\left(\ln \frac{\varepsilon_{0}}{\varepsilon g_{r}}-1.71\right)\right], \quad \mu=\int_{0}^{\infty} e^{-2 x / \eta_{1}} \chi(x) \frac{d x}{\eta_{1}} .
$$

It should be noted that found Eq.(17) has a good accuracy only for energy much smaller (at least on one order of magnitude) than $\varepsilon_{0}$ (see discussion after Eq. $(15)$ in $^{15}$ ).

\subsection{Pair creation}

For small value of the parameter $\kappa$ the probability of coherent pair creation is (see Eq.(12.11) in ${ }^{8}$ )

$$
W^{F}=\frac{9}{32} \sqrt{\frac{\pi}{2}} \frac{\alpha m^{2}}{\omega x_{0}} \frac{\kappa_{m}^{2}}{\sqrt{-\kappa_{m}^{\prime \prime}}} \exp \left(-8 / 3 \kappa_{m}\right)
$$


where $\kappa_{m}$ (which defines the value of $\omega_{t}$ ) is given in Eq. $(6), \kappa_{m}^{\prime \prime}=\kappa^{\prime \prime}\left(x_{m}\right)$. We find that $\omega_{t} \sim \omega_{m} \sim \omega_{0}$ for main axes of crystals of heavy elements. So at $\omega \sim \omega_{t}$ all the discussed effects are simultaneously essential in these crystals. In crystals of elements with intermediate $Z$ (Ge, Si, diamond) the ratio $\omega_{t} / \omega_{m} \sim 1$ but $\omega_{m} / \omega_{0} \ll 1$. So, the LPM effect for such crystals is significantly weaker.

At $\omega \ll \omega_{t}$ the incoherent mechanism of pair creation dominates. It integral cross section in oriented crystal has the form (see Eq.(26.30) in $^{8}$ )

$$
\sigma_{p}=\frac{28 Z^{2} \alpha^{3}}{9 m^{2}}\left[L_{0}-\frac{1}{42}-h\left(\frac{u_{1}^{2}}{a^{2}}\right)\right]
$$

where notations see in Eq.(9).

The influence of axis field on the incoherent pair creation process begins when $\omega$ becomes close to $\omega_{m}$. For small values of the parameter $\kappa_{m}$ the correction to the cross section Eq.(6) is (see Eq.(7.137) in ${ }^{8}$ )

$$
\Delta \sigma_{p}=\frac{176}{175} \frac{Z^{2} \alpha^{3}}{m^{2}} \overline{\kappa^{2}}\left(L_{u}-\frac{1789}{1980}\right), \quad \overline{\kappa^{2}}=\int_{0}^{\infty} \frac{d x}{\eta_{1}} e^{-x / \eta_{1}} \kappa^{2}(x), \quad L_{u}=L_{0}-h\left(\frac{u_{1}^{2}}{a^{2}}\right)
$$

The coherent and incoherent contribution to pair creation can separated also for $\kappa_{m} \gg 1\left(\omega \gg \omega_{m}\right)$. In this case one can use the perturbation theory in calculation of the probability of incoherent process and neglect the LPM effect because of domination of the coherent contribution and additional suppression (by the axis field) the incoherent process. In this case the local cross section of pair creation has the form (see Eq.(7.138) in ${ }^{8}$ )

$$
\sigma_{p}(x)=\frac{8 Z^{2} \alpha^{3} \Gamma^{3}(1 / 3)}{25 m^{2}(3 \kappa(x))^{2 / 3} \Gamma(2 / 3)}\left(L_{u}+0.4416+\frac{1}{3} \ln \kappa(x)\right) .
$$

Averaging the function $(\kappa(x))^{-2 / 3}$ and $\ln \kappa(x)(\kappa(x))^{-2 / 3}$ over $x$ according with Eq.(20) one can find the effective value of upper boundary of the transverse momentum transfer $\left(\propto m \kappa_{m}^{1 / 3}\right.$ instead of $\left.m\right)$ which contributes to the value $<\mathbf{q}_{s}^{2}>$. Using the obtained results we determine the effective logarithm $L$ by means of interpolation procedure

$$
L=L_{0} g, \quad g=1+\frac{1}{L_{0}}\left[-\frac{1}{42}-h\left(\frac{u_{1}^{2}}{a^{2}}\right)+\frac{1}{3} \ln \left(\frac{6-3 \kappa_{m}^{2}+3 \kappa_{m}^{3}}{6+\kappa_{m}^{2}}\right)\right] .
$$

Let us introduce the local characteristic energy of photon

$$
\omega_{c}(x)=\frac{m}{4 \pi Z^{2} \alpha^{2} \lambda_{c}^{3} n(x) L}=\frac{\omega_{e}\left(n_{a}\right)}{\xi(x) g}=\frac{\omega_{0}}{g} e^{x / \eta_{1}}
$$

where $\lambda_{c}=1 / m$. In this notations the local probability for small values of $\kappa_{m}$ and $\omega / \omega_{0}$ has a form (see Eq.(7.137) in ${ }^{8}$ and Eq.(2.23) in $\left.^{14}\right)$

$$
W(x)=\frac{7}{9 \pi} \frac{\alpha m^{2}}{\omega_{c}(x)}\left[1+\frac{396}{1225} \kappa^{2}(x)-\frac{3312}{2401} \frac{\omega^{2}}{\omega_{c}^{2}(x)}\right],
$$

where the term with $\kappa^{2}(x)$ arises due to the field action and the term with $\omega^{2} / \omega_{c}^{2}(x)$ reflects influence of multiple scattering (the LPM effect). Averaging this expression over $x$ we have

$$
\begin{aligned}
& \int_{0}^{\infty} \frac{d x}{x_{0}} \frac{1}{\omega_{c}(x)}=\frac{g}{\omega_{0}} \frac{\eta_{1}}{x_{0}}=\frac{g}{\omega_{e}\left(n_{a}\right)}, \quad \int_{0}^{\infty} \frac{d x}{x_{0}} \frac{1}{\omega_{c}^{3}(x)}=\frac{g}{\omega_{e}\left(n_{a}\right)} \frac{g^{2}}{3 \omega_{0}^{2}}, \\
& \int_{0}^{\infty} \frac{d x}{x_{0}} \frac{\kappa^{2}(x)}{\omega_{c}(x)}=\frac{g}{\omega_{e}\left(n_{a}\right)} \overline{\kappa^{2}}, \quad W \equiv \overline{W(x)}=W_{0} g\left[1+\frac{396}{1225} \overline{\kappa^{2}}-\frac{1104}{2401}\left(\frac{\omega g}{\omega_{0}}\right)^{2}\right],
\end{aligned}
$$


where $W_{0}$ is

$$
W_{0}=\frac{7}{9} \frac{\alpha m^{2}}{\pi \omega_{e}\left(n_{a}\right)}=\frac{28}{9} \frac{Z^{2} \alpha^{3}}{m^{2}} n_{a} L_{0} .
$$

\section{GENERAL THEORY}

\subsection{Radiation}

The spectral probability of radiation under the simultaneous action of multiple scattering and an external constant field was derived in ${ }^{8}$ (see Eqs.(7.89) and (7.90)). Multiplying the expression by $\omega$ and integrating over $\omega$ one obtains the total intensity of radiation $I$. For further analysis and numerical calculation it is convenient to carry out some transformations

1. Changing of variables: $v \rightarrow a v / 2, \tau \rightarrow 2 t / a,(v \tau \rightarrow v t)$.

2. Turn the contour of integration over $t$ at the angle $-\pi / 4$.

One finds after substitution $t \rightarrow \sqrt{2} t$

$$
\begin{aligned}
& I(\varepsilon)=\frac{\alpha m^{2}}{2 \pi} \int_{0}^{1} \frac{y d y}{1-y} \int_{0}^{x_{0}} \frac{d x}{x_{0}} G_{r}(x, y), \quad G_{r}(x, y)=\int_{0}^{\infty} F_{r}(x, y, t) d t-r_{3} \frac{\pi}{4}, \quad y=\frac{\omega}{\varepsilon}, \\
& F_{r}(x, y, t)=\operatorname{Im}\left\{e^{\varphi_{1}(t)}\left[r_{2} v_{0}^{2}\left(1+i b_{r}\right) \varphi_{2}(t)+r_{3} \varphi_{3}(t)\right]\right\}, \quad b_{r}=\frac{4 \chi^{2}(x)}{u^{2} v_{0}^{2}}, \quad u=\frac{y}{1-y} \\
& \varphi_{1}(t)=(i-1) t+b_{r}(1+i)\left(\varphi_{2}(t)-t\right), \quad \varphi_{2}(t)=\frac{\sqrt{2}}{v_{0}} \tanh \frac{v_{0} t}{\sqrt{2}}, \quad \varphi_{3}(t)=\frac{\sqrt{2} v_{0}}{\sinh \left(\sqrt{2} v_{0} t\right)}
\end{aligned}
$$

where

$$
r_{2}=1+(1-y)^{2}, \quad r_{3}=2(1-y), \quad v_{0}^{2}=\frac{1-y}{y} \frac{\varepsilon}{\varepsilon_{c}(x)},
$$

$\omega$ is the photon energy, the function $\varepsilon_{c}(x)$ is defined in Eq.(15) and $\chi(x)$ is defined in Eq.(5).

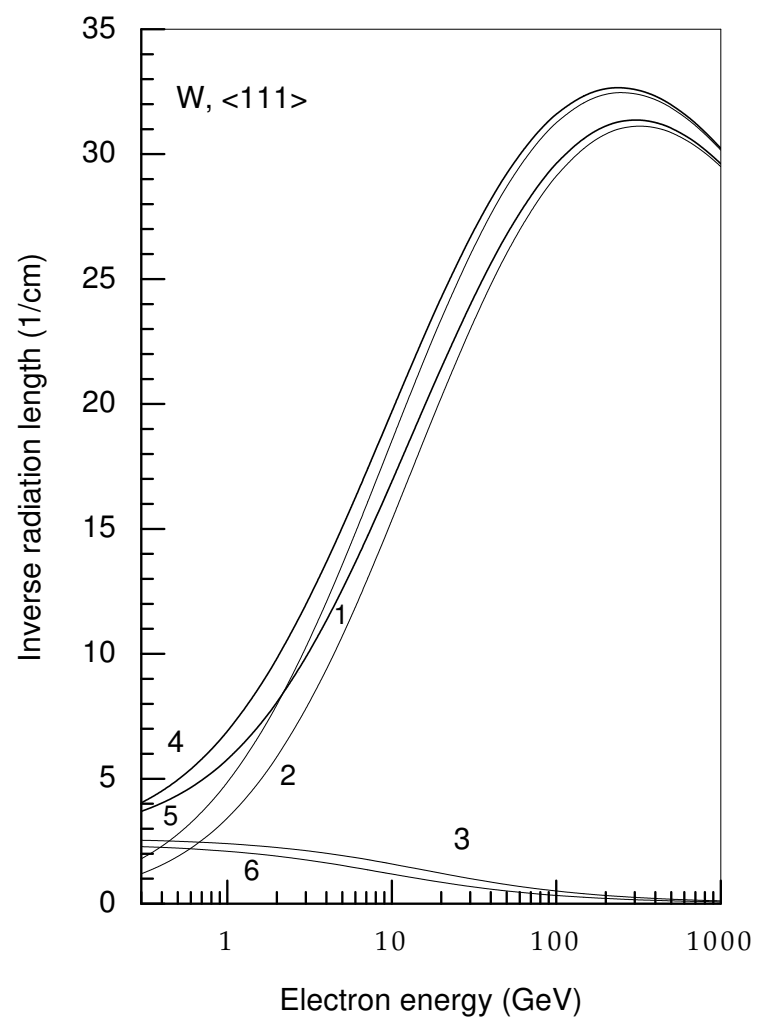

Figure 1: The inverse radiation length in tungsten, axis $<111>$ at different temperatures $\mathrm{T}$ vs the electron initial energy. Curves 1 and 4 are the total effect: $L^{c r}(\varepsilon)^{-1}=I(\varepsilon) / \varepsilon$ Eq.(27) for $\mathrm{T}=293 \mathrm{~K}$ and $\mathrm{T}=100$ $K$ correspondingly, the curves 2 and 5 give the coher-
In order to single out the influence of the multiple scattering (the LPM effect) on the process under consideration, we should consider both the coherent and incoherent contributions. The probability of coherent radiation is the first term $\left(v_{0}^{2}=0\right)$ of the decomposition of Eq.(27) over $v_{0}^{2}$. The coherent intensity of radiation is (compare with Eq.(17.7) in $^{8}$ )

$$
I^{F}(\varepsilon)=\int_{0}^{x_{0}} I(\chi) \frac{d x}{x_{0}} .
$$

Here $I(\chi)$ is the radiation intensity in constant field (magnetic bremsstrahlung limit, see Eqs. (4.50), (4.51) in ${ }^{8}$ ). It is convenient to use the following representation for $I(\chi)$

$$
\begin{aligned}
& I(\chi)=i \frac{\alpha m^{2}}{2 \pi} \int_{\lambda-i \infty}^{\lambda+i \infty}\left(\frac{\chi^{2}}{3}\right)^{s} \Gamma(1-s) \Gamma(3 s-1) \\
& \times(2 s-1)\left(s^{2}-s+2\right) \frac{d s}{\cos \pi s}, \quad \frac{1}{3}<\lambda<1 .
\end{aligned}
$$


The intensity of incoherent radiation is the second term $\left(\propto v_{0}^{2}\right)$ of the mentioned decomposition. The expression for the intensity of incoherent radiation follows from Eq.(21.21) in ${ }^{8}$ ):

$$
I^{i n c}(\varepsilon)=\frac{\alpha m^{2}}{60 \pi} \frac{\varepsilon}{\varepsilon_{0}} g \int_{0}^{x_{0}} e^{-x / \eta_{1}} J(\chi) \frac{d x}{x_{0}},
$$

here $J(\chi)$ is the integral over photon energy $\omega$ :

$$
\begin{aligned}
& J(\chi)=\int_{0}^{1}\left[y^{2}\left(f_{1}(z)+f_{2}(z)\right)+2(1-y) f_{2}(z)\right] d y, \\
& z=\left(\frac{y}{\chi(1-y)}\right)^{2 / 3}
\end{aligned}
$$

where $y=\omega / \varepsilon$, the functions $f_{1}(z)$ and $f_{2}(z)$ are defined in the just mentioned equation in: ${ }^{8}$

$$
\begin{aligned}
& f_{1}(z)=z^{4} \Upsilon(z)-3 z^{2} \Upsilon^{\prime}(z)-z^{3}, \\
& f_{2}(z)=\left(z^{4}+3 z\right) \Upsilon(z)-5 z^{2} \Upsilon^{\prime}(z)-z^{3},
\end{aligned}
$$

here $\Upsilon(z)$ is the Hardy function:

$$
\Upsilon(z)=\int_{0}^{\infty} \sin \left(z \tau+\frac{\tau^{3}}{3}\right) d \tau .
$$

We used the following relations between the function $\Upsilon(z)$ and its derivatives:

$$
\begin{aligned}
& \Upsilon^{(n)}=\frac{d^{n}}{d z^{n}} \operatorname{Im} \int_{0}^{\infty} \exp \left(i\left(z \tau+\frac{\tau^{3}}{3}\right)\right) d \tau=\operatorname{Im} \int_{0}^{\infty}(i \tau)^{n} \exp \left(i\left(z \tau+\frac{\tau^{3}}{3}\right)\right) d \tau, \\
& z \Upsilon(z)=\Upsilon^{\prime \prime}(z)+1, \quad \Upsilon^{(n+3)}(z)=(n+1) \Upsilon^{(n)}+z \Upsilon^{(n+1)} .
\end{aligned}
$$

Integrating Eq.(32) by parts one can represent the integral $J(\chi)$ in the form

$$
\begin{aligned}
& J(\chi)=\frac{\chi^{3}}{6} \frac{d^{2}}{d \chi^{2}}\left(J_{1}(\chi)+J_{2}(\chi)\right)+\frac{d}{d \chi}\left(\chi^{2} J_{2}(\chi)\right), \quad J_{1,2}(\chi)=\frac{f_{1,2}(\infty)}{\chi}+i_{1,2}(\chi), \\
& i_{1,2}(\chi)=\chi \int_{0}^{\infty} f_{1,2}^{\prime}(z) \frac{z^{3} d z}{1+\chi z^{3 / 2}} . \quad f_{1}^{\prime}(z)=z^{2} \Upsilon^{(5)}(z)-3 z \Upsilon^{(4)}, \quad f_{2}^{\prime}(z)=z^{2} \Upsilon^{(5)}(z)-5 z \Upsilon^{(4)}+3 \Upsilon^{(3)} .
\end{aligned}
$$

Since the integrals in Eq.(36) for the separate terms of functions $f_{1.2}^{\prime}(z)$ in form Eq.(33) diverges, we transformed it to the form Eq.(36). We used also the important formula

$$
\int_{0}^{\infty} z^{3 / 2} f_{1,2}^{\prime}(z) d z=0
$$

which follows from the equation $\int_{0}^{\infty} \Upsilon^{\prime}(z) \frac{d z}{\sqrt{z}}=0$ having applied integration by parts for separate terms of functions $f_{1.2}^{\prime}(z)$ in form Eq.(36). 
Entering in Eq.(36) expression $(1+u)^{-1}$ we present as contour integral

$$
\frac{1}{(1+u)}=\frac{i}{2} \int_{\lambda-i \infty}^{\lambda+i \infty} \frac{u^{s}}{\sin \pi s} d s, \quad u=\chi z^{3 / 2}, \quad-1<\lambda<0 .
$$

Substituting in the integral in Eq.(36) the functions $f_{1,2}^{\prime}(z)$ in the form given by the same equation and integrating over the variables $z$ and $\tau$, we get after change of variable $s \rightarrow 2 s$, displacement of integration contour and reduction of similar terms the new representation of the function $J(\chi)$, which is suitable for both analytical and numerical calculation:

$$
J(\chi)=\frac{i \pi}{2} \int_{\lambda-i \infty}^{\lambda+i \infty} \frac{\chi^{2 s}}{3^{s}} \frac{\Gamma(1+3 s)}{\Gamma(s)} R(s) \frac{d s}{\sin ^{2} \pi s}, \quad-\frac{1}{3}<\lambda<0
$$

where

$$
R(s)=15+43 s+31 s^{2}+28 s^{3}+12 s^{4} .
$$

In the case $\chi \ll 1$, closing the integration contour on the right, one can calculate the asymptotic series in powers of $\chi$

$$
J(\chi)=15+516 \chi^{2}\left(\ln \frac{\chi}{\sqrt{3}}-C\right)+1893 \chi^{2}+\ldots \simeq 15\left[1-34.4 \chi^{2}\left(\ln \frac{1}{\chi}-2.542\right)\right]
$$

Closing the integration contour on the left one obtains the series over the inverse powers of $\chi$

$$
J(\chi)=\frac{58 \pi \Gamma(1 / 3)}{81 \cdot 3^{1 / 6} \chi^{2 / 3}}+\frac{628 \pi 3^{1 / 6} \Gamma(2 / 3)}{243 \chi^{4 / 3}}-\frac{13}{\chi^{2}}\left(\ln \chi-\frac{1}{2} \ln 3-C+\frac{57}{52}\right)+\ldots
$$

Now we get over to the third term $\left(\propto v_{0}^{4}\right)$ of the decomposition. In this case it is convenient to turn back the integration contour in Eq.(27) and perform inverse transformation $t \rightarrow t / \sqrt{2}$, so that $\sqrt{2} v_{0} t \rightarrow v t\left(v=\sqrt{i} v_{0}\right)$. In the terms $\propto v_{0}^{4}$ of the decomposition the integrals over $t$ have the form

$$
\int_{0}^{\infty} \exp \left(-i\left(t+\frac{a t^{3}}{3}\right)\right) t^{2 n+1} d t, \quad \text { or } \quad i \int_{0}^{\infty} \exp \left(-i\left(t+\frac{a t^{3}}{3}\right)\right) t^{2 n} d t
$$

where $a=\chi^{2} / u^{2}$. The radiation intensity is contains the imaginary part of these integrals where the integrand is even function of $t$. Because of this the final result is expressed in terms of MacDonald functions $K_{1 / 3}(z)$ and $K_{2 / 3}(z)(z=2 u / 3 \chi)$ and their derivatives. Using the recurrence relations we find after quite cumbersome calculation

$$
I^{(3)}=-\frac{\alpha m^{2}}{8400} \frac{\varepsilon^{2}}{\varepsilon_{0}^{2}} g_{r}^{2} \int_{0}^{x_{0}} e^{-2 x / \eta_{1}} P(\chi) \frac{d x}{x_{0}},
$$

where

$$
\begin{aligned}
& P(\chi)=\frac{9 \sqrt{3}}{64 \pi} \int_{0}^{1}\left[r_{2} F_{2}(z)+r_{3} F_{3}(z)\right] z^{3} \frac{1-y}{y} d y, \quad u=\frac{y}{1-y} \\
& F_{2}(z)=\left(7820+126 z^{2}\right) z K_{2 / 3}(z)-\left(280+2430 z^{2}\right) K_{1 / 3}(z), \quad F_{3}(z)=\left(264-63 z^{2}\right) z K_{2 / 3}(z)-\left(24+3 z^{2}\right) K_{1 / 3}(z) .
\end{aligned}
$$

Passing on to the variable $u$ and having applied the representation

$$
\frac{1}{(1+u)^{m}}=\frac{1}{2 \pi i} \int_{\lambda-i \infty}^{\lambda+i \infty} \frac{\Gamma(-s) \Gamma(m+s)}{\Gamma(m)} u^{s} d s, \quad-m<\lambda<0
$$


taking into account the table integrals over $u$

$$
\int_{0}^{\infty} x^{\mu} K_{v}(x) d x=2^{\mu-1} \Gamma\left(\frac{1+\mu+v}{2}\right) \Gamma\left(\frac{1+\mu-v}{2}\right),
$$

substituting $s \rightarrow 2 s$ and using the tripling formula

$$
3^{3 s} \Gamma(s) \Gamma(s+1 / 3) \Gamma(s+2 / 3)=2 \pi \sqrt{3} \Gamma(3 s),
$$

we get after reduction of similar terms the following expression for the function $P(\chi)$

$$
\begin{aligned}
& P(\chi)=\frac{1}{2 \pi i} \int_{\lambda-i \infty}^{\lambda+i \infty} D(s)\left(\frac{\chi^{2}}{3}\right)^{s-1}(1-2 s) \Gamma(1-s) \Gamma(3 s) \frac{d s}{\cos (\pi s)}, \quad 0<\lambda<1, \\
& D(s)=192+532 s-210 s^{2}+73 s^{3}-349 s^{4}+42 s^{5} .
\end{aligned}
$$

Closing the integration contour to the right we get the asymptotic series over powers of $\chi$

$$
P(\chi)=560+5985 \sqrt{3} \chi-388800 \chi^{2}+\ldots
$$

Closing the integration contour to the left we get the series over powers of $1 / \chi$

$$
P(\chi)=\frac{192}{\chi^{2}}+\frac{4280}{243} 3^{1 / 3} \Gamma\left(\frac{1}{3}\right) \frac{1}{\chi^{8 / 3}}-\frac{635 \sqrt{3}}{\chi^{3}}-+\ldots
$$

The inverse radiation length in tungsten crystal (axis $<111>$ ) $1 / L^{c r}(\varepsilon)=I(\varepsilon) / \varepsilon$ Eq.(27), well as coherent contribution $1 / L^{F}(\varepsilon)=I^{F}(\varepsilon) / \varepsilon$ Eq.(29) and incoherent contribution $1 / L^{i n c}(\varepsilon)=I^{i n c}(\varepsilon) / \varepsilon$ Eq.(31) are shown in Fig.1 for two temperatures $\mathrm{T}=100 \mathrm{~K}$ and $\mathrm{T}=293 \mathrm{~K}$ as a function of incident electron energy $\varepsilon$. In low energy region $(\varepsilon \leq 0.3 \mathrm{GeV})$ the asymptotic expressions Eqs.(7) and (8) are valid. One can see that at temperature T=293 K the intensity $I^{F}(\varepsilon)$ is equal to $I^{\text {inc }}(\varepsilon)$ at $\varepsilon \simeq 0.4 \mathrm{GeV}$ and temperature $\mathrm{T}=100 \mathrm{~K}$ the intensity $I^{F}(\varepsilon)$ is equal to $I^{\text {inc }}(\varepsilon)$ at $\varepsilon \simeq 0.7 \mathrm{GeV}$. The same estimates follow from comparison of Eqs.(7) and (8), see also Eq.(10). At higher energies the intensity $I^{F}(\varepsilon)$ dominates while the intensity $I^{\text {inc }}(\varepsilon)$ decreases monotonically.

The inverse radiation length given in Fig.1 can be compared with data directly only if the crystal thickness $l \ll L^{c r}(\varepsilon)$ (thin target). Otherwise one has to take into account the energy loss. The corresponding analysis is simplified essentially if $l \leq L^{\min }=(\max (I(\varepsilon) / \varepsilon))^{-1}$. The radiation length $L^{c r}(\varepsilon)$ varies slowly on the electron trajectory for such thicknesses. This is because of weak dependence of $L^{c r}(\varepsilon)$ on energy in the region $L^{c r}(\varepsilon) \simeq L^{\min }$ and the relatively large value of $L^{c r}(\varepsilon) \gg L^{\min }$ in the region where this dependence is essential but variation of energy on the thickness $l$ is small. For $\mathrm{W}$, axis $<111>$, T=293 $\mathrm{K}$ one has $L^{\mathrm{min}}=320 \mu \mathrm{m}$ at energy $\varepsilon=300 \mathrm{GeV}$, see Fig.1. For this situation dispersion can be neglected (see discussion in Sec.17.5 of ${ }^{8}$ ) and energy loss equation acquires the form

$$
\frac{1}{\varepsilon} \frac{d \varepsilon}{d l}=-L^{c r}(\varepsilon)^{-1} \equiv-\frac{I(\varepsilon)}{\varepsilon} .
$$

In the first approximation the final energy of electron is

$$
\varepsilon_{1}=\varepsilon_{0} \exp \left(-l / L^{c r}\left(\varepsilon_{0}\right)\right),
$$

where $\varepsilon_{0}$ is the initial energy. In the next approximation one has

$$
\ln \frac{\varepsilon(l)}{\varepsilon_{0}}=-L^{c r}\left(\varepsilon_{0}\right) \int_{\varepsilon_{1}}^{\varepsilon_{0}} L^{c r}(\varepsilon)^{-1} \frac{d \varepsilon}{\varepsilon} .
$$


(a)

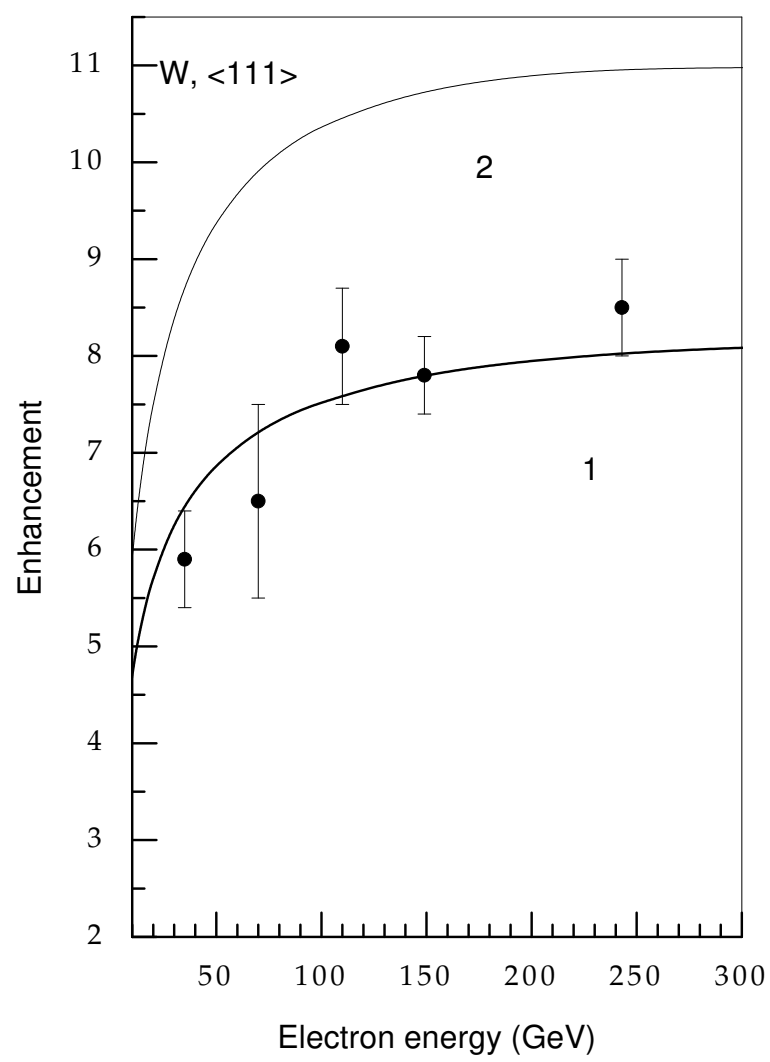

(b)
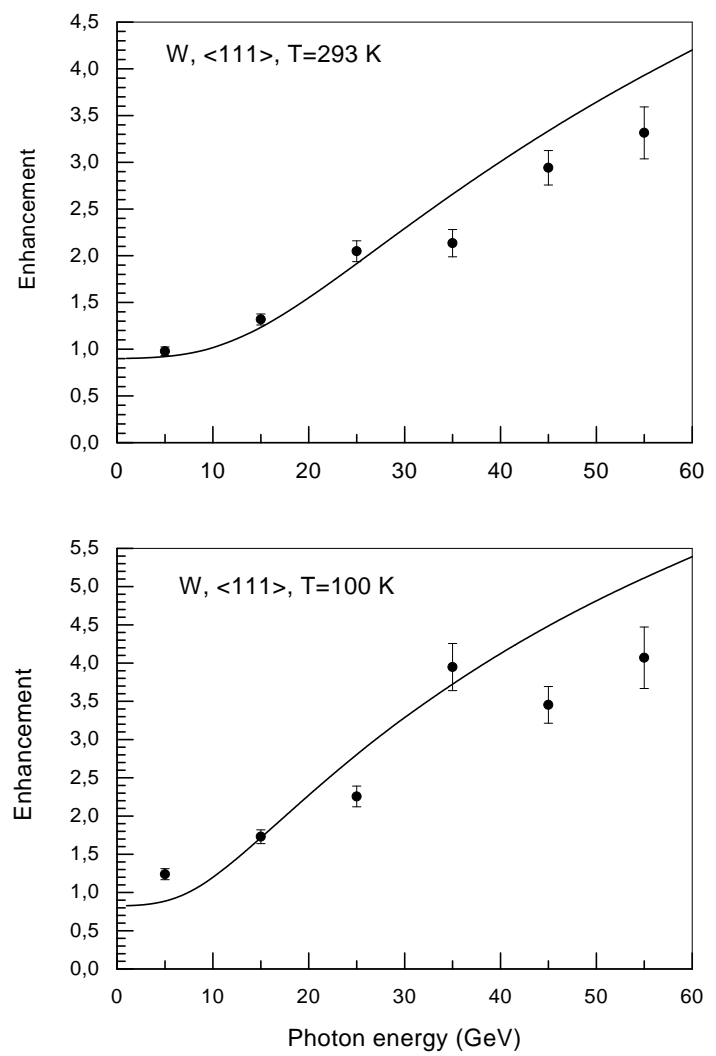

Figure 2: Comparison of theory and experiment.(a) Enhancement of radiation intensity (the ratio $L^{B M} / L^{e f}$ ) in tungsten, axis $<111>, \mathrm{T}=293 \mathrm{~K}$. The curve 1 is for the target with thickness $l=200 \mu \mathrm{m}$, where the energy loss was taken into account (according with Eq.(56)). The curve 2 is for a considerably more thinner target, where one can neglect the energy loss $\left(L^{e f} \rightarrow\right.$ $\left.L^{c r}\right)$. The data are from. ${ }^{10}$

(b) Enhancement of the probability of pair creation in tungsten for different temperatures, axis $\langle 111\rangle$. The data are from. ${ }^{12}$

If the dependence of $L^{c r}(\varepsilon)^{-1}$ on $\varepsilon$ is enough smooth it's possible to substitute the function $L^{c r}(\varepsilon)^{-1}$ by an average value with the weight $1 / \varepsilon$ :

$$
L^{c r}(\varepsilon)^{-1} \rightarrow \frac{\varepsilon_{0} L^{c r}\left(\varepsilon_{1}\right)^{-1}+\varepsilon_{1} L^{c r}\left(\varepsilon_{0}\right)^{-1}}{\varepsilon_{0}+\varepsilon_{1}} \equiv \frac{1}{\bar{L}}
$$

Numerical test confirms this simplified procedure. Using it we find

$$
\ln \frac{\varepsilon(l)}{\varepsilon_{0}}=-\frac{L^{c r}\left(\varepsilon_{0}\right)}{\bar{L}} \ln \frac{\varepsilon_{0}}{\varepsilon_{1}}=-\frac{l}{\bar{L}}, \quad \frac{\Delta \varepsilon}{\varepsilon_{0}}=1-\exp \left(-\frac{l}{\bar{L}}\right) \equiv \frac{l}{L^{e f}} .
$$

Enhancement of radiation length (the ratio of Bethe-Maximon radiation length $L^{B M}$ and $L^{e f}$ ) in tungsten, axis $<111>$, $\mathrm{T}=293 \mathrm{~K}$ is shown in Fig.2(a). The curve 1 is for the target with thickness $l=200 \mu m$, where the energy loss was taken into account according using the simplified procedure Eq.(56). The curve 2 is for a considerably more thinner target, where one can neglect the energy loss. The only available data are from. ${ }^{10}$ The measurement of radiation from more thin targets is of evident interest.

\subsection{Pair creation}

The general expression for integral probability of pair creation by a photon under the simultaneous action of multiple scattering and an external constant field was obtained in ${ }^{5}$ (see Eqs.(2.14) and (1.12)). This expression can be found also from 
Eq.(27) using the standard QED substitution rules: $\varepsilon \rightarrow-\varepsilon, \omega \rightarrow-\omega, \omega^{2} d \omega \rightarrow \varepsilon^{2} d \varepsilon$ and exchange $\varepsilon_{c}(x) \rightarrow \omega_{c}(x) / 4$;

$$
\begin{aligned}
& W=\frac{\alpha m^{2}}{2 \pi \omega} \int_{0}^{1} \frac{d y}{y(1-y)} \int_{0}^{x_{0}} \frac{d x}{x_{0}} G(x, y), \quad G(x, y)=\int_{0}^{\infty} F(x, y, t) d t+s_{3} \frac{\pi}{4}, \\
& F(x, y, t)=\operatorname{Im}\left\{e^{f_{1}(t)}\left[s_{2} v_{0}^{2}(1+i b) f_{2}(t)-s_{3} f_{3}(t)\right]\right\}, \quad b=\frac{4 \kappa_{1}^{2}}{v_{0}^{2}}, \quad y=\frac{\varepsilon}{\omega}, \\
& f_{1}(t)=(i-1) t+b(1+i)\left(f_{2}(t)-t\right), \quad f_{2}(t)=\frac{\sqrt{2}}{v_{0}} \tanh \frac{v_{0} t}{\sqrt{2}}, \quad f_{3}(t)=\frac{\sqrt{2} v_{0}}{\sinh \left(\sqrt{2} v_{0} t\right)},
\end{aligned}
$$

where

$$
s_{2}=y^{2}+(1-y)^{2}, s_{3}=2 y(1-y), v_{0}^{2}=4 y(1-y) \frac{\omega}{\omega_{c}(x)}, \kappa_{1}=y(1-y) \kappa(x),
$$

$\varepsilon$ is the energy of one of the particles of pair, the function $\omega_{c}(x)$ is defined in Eq.(23) and $\kappa(x)$ is defined in Eq.(5).

(a)

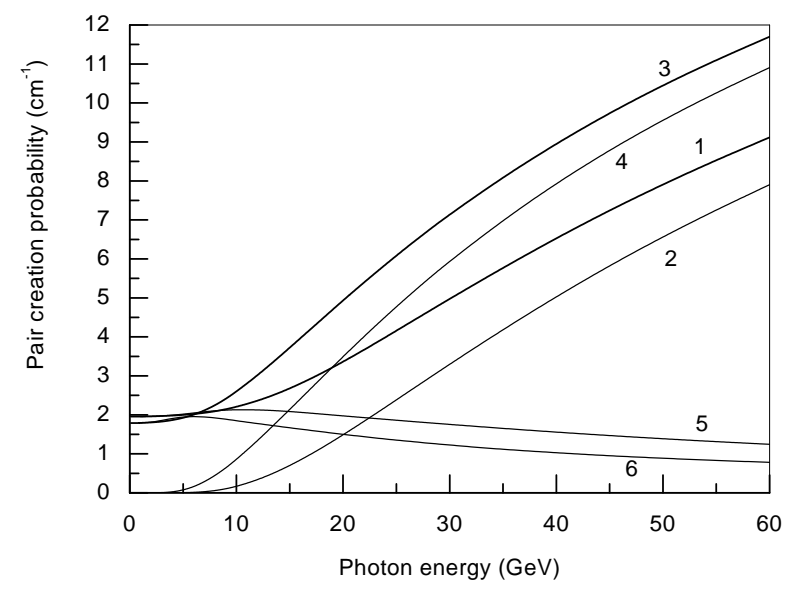

(b)

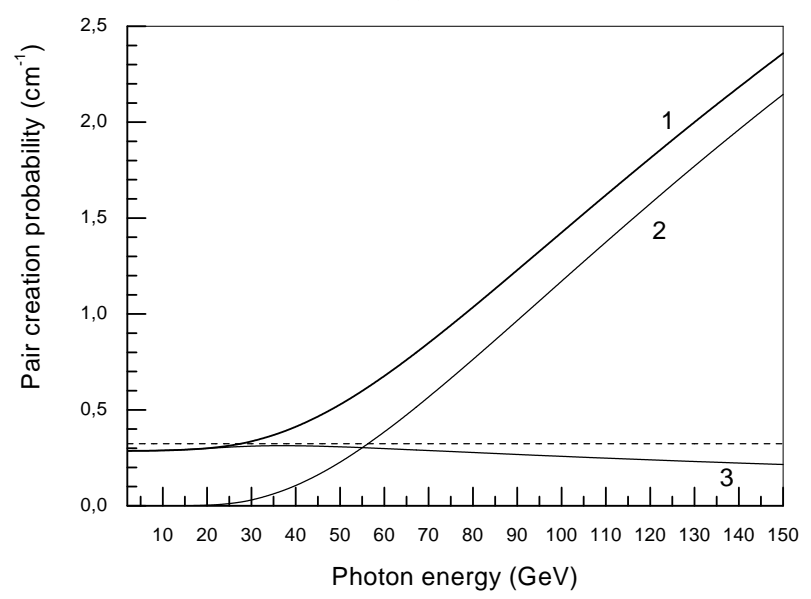

Figure 3: (a) Pair creation probability in tungsten, axis $<111>$ at different temperatures T. Curves 1 and 3 are the total probability $W$ Eq.(57) for T=293 $\mathrm{K}$ and $\mathrm{T}=100 \mathrm{~K}$, the curves 2 and 4 give the coherent contribution $W^{F}$ Eq.(59), the curves 5 and 6 give the incoherent contribution $W^{\text {inc }}$ Eq.(60) at corresponding temperatures T.

(b) Pair creation probability in germanium, axis $<110>$ at $\mathrm{T}=100 \mathrm{~K}$. Curve 1 is the total probability $W$ Eq.(57), the curve 2 gives the coherent contribution $W^{F}$ Eq.(59), the curve 3 gives the incoherent contribution $W^{i n c}$ Eq.(60). The dashed line is the Bethe-Maximon probability

In order to single out the influence of the multiple scattering (the LPM effect) on the process under consideration, we should consider both the coherent and incoherent contributions. The probability of coherent pair creation is the first term $\left(v_{0}^{2}=0\right)$ of the decomposition of Eq.(57) over $v_{0}^{2}$ (compare with Eq.(2.17) in ${ }^{5}$ and see Eq.(12.7) in ${ }^{8}$ )

$$
\begin{aligned}
& W^{F}=\frac{\alpha m^{2}}{2 \sqrt{3} \pi \omega} \int_{0}^{1} \frac{d y}{y(1-y)} \int_{0}^{x_{0}} \frac{d x}{x_{0}}\left[2 s_{2} K_{2 / 3}(\lambda)\right. \\
& \left.+s_{3} \int_{\lambda}^{\infty} K_{1 / 3}(z) d z\right], \quad \lambda=\frac{2}{3 \kappa_{1}} .
\end{aligned}
$$


The probability of incoherent pair creation is the second term $\left(\propto v_{0}^{2}\right)$ of the mentioned decomposition (compare with Eq.(2.26) in ${ }^{5}$ and compare with Eq.(21.31) in $\left.{ }^{8}\right)$

$$
W^{i n c}=\frac{4 Z^{2} \alpha^{3} n_{a} L}{15 m^{2}} \int_{0}^{1} d y \int_{0}^{\infty} \frac{d x}{\eta_{1}} e^{-x / \eta_{1}} f(x, y),
$$

where $L$ is defined in Eq.(10),

$$
f(x, y)=f_{1}(z)+s_{2} f_{2}(z), \quad z=z(x, y)=\kappa_{1}^{-2 / 3},
$$

here functions $f_{1,2}(z)$ are defined in Eq.(33). In further analysis and numerical calculation it is convenient to use given above representations of the Hardy function and its derivative Eqs.(34),(35).

The probabilities $W$ Eq.(57), $W^{F}$ Eq.(59), and $W^{\text {inc }}$ Eq.(60) at different temperatures T are shown in Fig.3 as a function of photon energy $\omega$. In low energy region $(\omega \leq 1 \mathrm{GeV})$ one can neglect the coherent process probability $W^{F}$ as well as influence of axis field on the incoherent process probability and the LPM effect and the probability of process is $W^{L E}=n_{a} \sigma_{p}$ Eq.(19). In this energy region as one can see in Fig. 3 the probability $W$ is by $10 \%$ at $\mathrm{T}=293 \mathrm{~K}$ and by $20 \%$ at $\mathrm{T}=100 \mathrm{~K}$ less than the probability at random orientation $W^{r a n}$ which is taken as $W^{r a n}=W^{B M}$ (the Bethe-Maximon probability is $W^{B M}=W_{0}\left(1-1 / 42 L_{0}\right)=2.17$ $1 / \mathrm{cm}$ in tungsten).

With energy increase the influence of axis field begins and the LPM effect manifests itself according to Eq.(25) (the terms with $\overline{\kappa^{2}}$ and $\left(\omega g / \omega_{0}\right)^{2}$ correspondingly). This leads first to not large increase of the probability $W^{\text {inc }}$ which attains the maximum at $\omega \sim \omega_{m}$. The probability $W^{F}$ in this region is defined by Eq.(18) and its contribution is relatively small. The probability $W^{F}$ becomes comparable with $W^{i n c}$ at $\omega \simeq 1.5 \omega_{m}$. At higher energies $W^{F}$ dominates, while $W^{\text {inc }}$ decreases monotonically.

In Fig.2(b) the calculated total integral probability $W$ of pair creation by a photon Eq.(57) is compared with data of NA43 CERN experiment. ${ }^{12}$ The enhancement is the ratio $W / W^{B M}$. One can see that the theory quite satisfactory describes data. This statement differs from conclusion made in. ${ }^{12}$ One of reasons for this difference is diminishing of incoherent contribution (see Fig.3): for $\mathrm{W},<111>, \mathrm{T}=100 \mathrm{~K}$ at photon energy $\omega=55 \mathrm{GeV}$ one has $W^{i n c}=0.35 W^{B M}$, while in ${ }^{12}$ it was assumed that $W^{\text {inc }}=W^{B M}$.

The third term $\left(\propto v_{0}^{4}\right)$ of the decomposition the pair creation probability over $v_{0}^{2}$ can be obtained from the corresponding expressions for radiation using the QED standard substitutions (cp with Eq.(57)) and taking into account that $\omega_{0}=4 \varepsilon_{0}$ (see Table 1)

$$
\begin{aligned}
& W^{(3)}=-\frac{\alpha m^{2}}{8400} \frac{\omega}{\omega_{0}^{2}} g^{2} \int_{0}^{x_{0}} e^{-2 x / \eta_{1}} T(\kappa) \frac{d x}{x_{0}}, \\
& T(\kappa)=\frac{9 \sqrt{3}}{4 \pi} \int_{0}^{1}\left[s_{2} F_{2}(\lambda)-s_{3} F_{3}(\lambda)\right] \lambda^{3} y(1-y) d y
\end{aligned}
$$

\section{THE LPM EFFECT IN ORIENTED CRYSTAL}

The contribution of the LPM effect in the total intensity of radiation $I$ Eq.(27) is defined as

$$
I^{L P M}=I-I^{F}-I^{i n c}
$$

The relative contribution (negative since the LPM effect suppresses the radiation process) $\Delta_{r}=-I^{L P M} / I$ is shown in Fig.4(a). This contribution has the maximum $\Delta_{r} \simeq 0.8 \%$ at $\varepsilon \simeq 0.7 \mathrm{GeV}$ for $\mathrm{T}=293 \mathrm{~K}$ and $\Delta_{r} \simeq 0.9 \%$ at $\varepsilon \simeq 0.3 \mathrm{GeV}$ for T=100 Kor, in general, at $\varepsilon \sim \varepsilon_{t}$. The left part of the curves is described quite satisfactory by Eq.(17). For explanation of the right part of the curves let us remind that at $\varepsilon \gg \varepsilon_{m}$ the behavior of the radiation intensity at $x \sim \eta_{1}$ is defined by the ratio of the contributions 
to the momentum transfer of multiple scattering and that of the external field on the formation length $l_{f}\left(\right.$ see Eq.(21.3) in $\left.{ }^{8}\right)$

$$
\begin{aligned}
& k=\frac{<\mathbf{q}_{s}^{2}>}{<\mathbf{q}>^{2}}=\frac{\dot{\vartheta}_{s}^{2} l_{f}}{\left(w l_{f}\right)^{2}} \sim \frac{\varepsilon}{\varepsilon_{0}} \chi_{m}^{-4 / 3}=\frac{\varepsilon}{\varepsilon_{0}}\left(\frac{\varepsilon_{m}}{\varepsilon}\right)^{4 / 3} \\
& \frac{1}{L^{F}} \sim \frac{\alpha}{l_{f}} \sim \frac{\alpha m^{2}}{\varepsilon} \chi_{m}^{2 / 3}=\frac{\alpha m^{2}}{\varepsilon_{m}} \chi_{m}^{-1 / 3}
\end{aligned}
$$

(a)

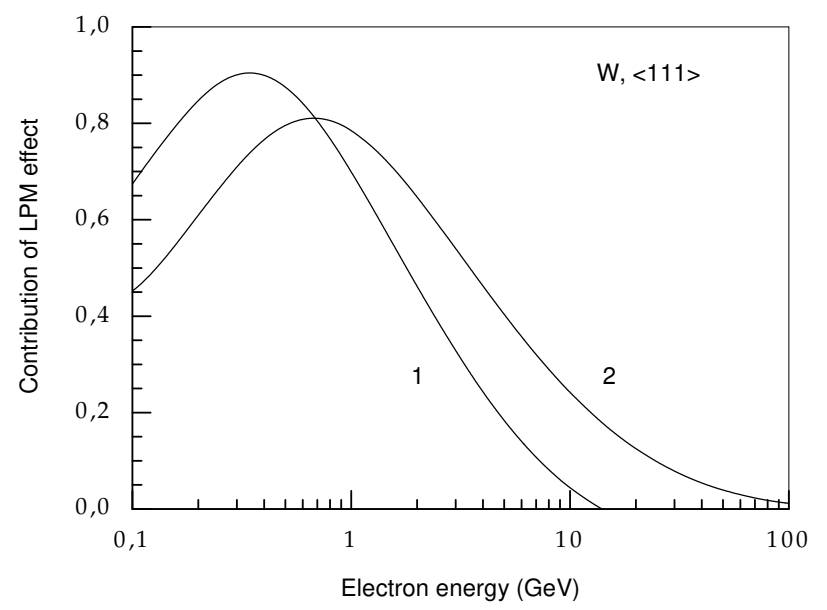

(b)

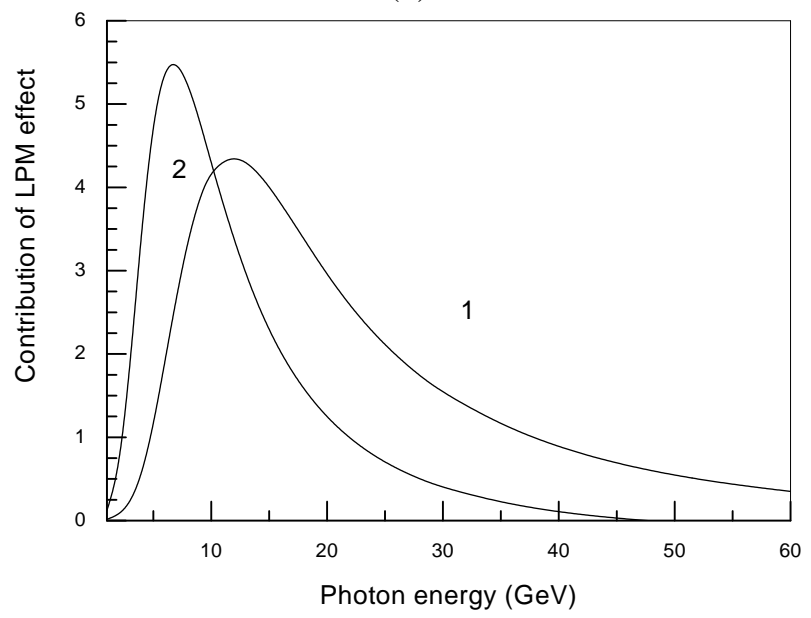

Figure 4: The LPM effect in oriented crystal:(a) The relative contribution into radiation intensity of the LPM effect $\Delta_{r}$ (per cent) in tungsten, axis $<111>$. Curve 1 is for $\mathrm{T}=100 \mathrm{~K}$ and curve 2 is for $\mathrm{T}=293 \mathrm{~K}$.

(b) The relative contribution of the LPM effect into total pair creation probability $\Delta$ (per cent) in tungsten, axis $<111>$. Curve 1 is for $\mathrm{T}=293 \mathrm{~K}$ and curve 2 is for $\mathrm{T}=100 \mathrm{~K}$.

where $w$ is an acceleration in an external field. The linear over $k$ term determines the contribution into intensity of incoherent process: $1 / L^{i n c}\left(\varepsilon \gg \varepsilon_{m}\right) \sim k / L^{F}(\varepsilon) \sim \alpha m^{2} /\left(\varepsilon_{0} \chi_{m}^{2 / 3}\right)$. The LPM effect is defined by the next term of decomposition over $k\left(\propto k^{2}\right)$ and decreases with energy even faster than $1 / L^{i n c}(\varepsilon)$. Moreover one has to take into account that at $\varepsilon \geq \varepsilon_{s}$ the contribution of relevant region $x \sim \eta_{1}$ into the total radiation intensity is small and $1 / L^{F}(\varepsilon)$ decreases with the energy growth as $\chi_{m}^{-1 / 3}$. For such energies the main contribution gives the region $x \sim \chi_{s}^{2 / 3}=\left(\varepsilon / \varepsilon_{s}\right)^{2 / 3}$ and $1 / L^{c r}(\varepsilon)$ increases until energy $\varepsilon \sim 10 \varepsilon_{s}$ (see Fig.1). This results in essential reduction of relative contribution of the LPM effect $\Delta_{r}$.

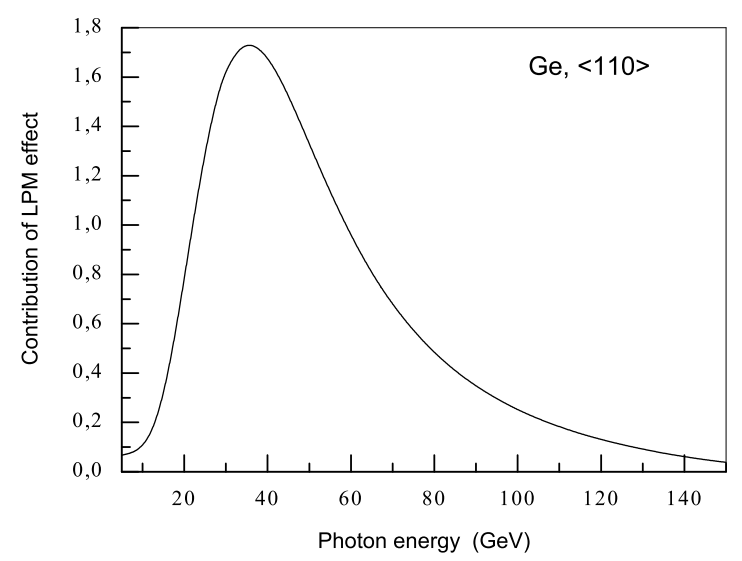

Figure 5: The relative contribution of the LPM effect into total pair creation probability $\Delta$ (per cent) in germanium crystal, axis $<110>$, for $\mathrm{T}=100 \mathrm{~K}$.
The contribution of the LPM effect in the total pair creation probability $W$ Eq.(57) is defined as

$$
W^{L P M}=W-W^{F}-W^{i n c}
$$

The relative contribution (negative since the LPM effect suppresses the process) $\Delta=-W^{L P M} / W$ is shown in Fig.4(b). This contribution has the maximum $\Delta \simeq 5.5 \%$ at $\omega \simeq 7 \mathrm{GeV}$ for $\mathrm{T}=293 \mathrm{~K}$ and $\Delta \simeq 4.3 \%$ at $\omega \simeq$ $12 \mathrm{GeV}$ for $\mathrm{T}=100 \mathrm{~K}$ for tungsten crystal. For germanium crystal the value $\Delta=-W^{L P M} / W$ is shown in Fig.5. This contribution attains the maximum $\Delta \simeq 1.7 \%$ at $\omega \simeq 34 \mathrm{GeV}$ for $\mathrm{T}=100 \mathrm{~K}$ or, in general, at $\omega \sim$ $\omega_{m}$. The left part of the curves is described by the term with $\left(\omega g / \omega_{0}\right)^{2}$ in Eq.(25). For understanding of the right part of the curves one has 
to take into account that at $\omega \gg \omega_{m}$ the behavior of the pair creation probability at $x \sim \eta_{1}$ is defined by the ratio of the contributions to the momentum transfer of multiple scattering and that of the external field on the formation length $l_{f}$ (see Eqs.(1.4), (2.28), (2.29) and discussion in $^{5}$ )

$$
\begin{aligned}
& k=\frac{\left\langle\mathbf{q}_{s}^{2}>\right.}{<\mathbf{q}>^{2}}=\frac{\dot{\vartheta}_{s}^{2} l_{f}}{\left(w l_{f}\right)^{2}} \sim \frac{\omega}{\omega_{0}} \kappa_{m}^{-4 / 3}=\frac{\omega}{\omega_{0}}\left(\frac{\omega_{m}}{\omega}\right)^{4 / 3} \\
& W^{F} \sim \frac{\alpha}{l_{f}} \sim \frac{\alpha m^{2}}{\omega} \kappa_{m}^{2 / 3}
\end{aligned}
$$

where $w$ is an acceleration in an external field. The linear over $k$ term determines the contribution into probability of incoherent process: $W^{\text {inc }}\left(\omega \gg \omega_{m}\right) \sim k W^{F} \sim \alpha m^{2} /\left(\omega_{0} \kappa_{m}^{2 / 3}\right)$ (cp Eq.(21)). The LPM effect is defined by the next term of decomposition over $k\left(\propto k^{2}\right)$ and decreases with energy even faster than $W^{i n c}$.

It follows from Eq.(66) that maximal influence of multiple scattering on the process under consideration is reached at $\omega \sim \omega_{m} \sim \omega_{0}$ where $k \sim 1$ for tungsten crystal. The analysis shows (see Fig.4 in ${ }^{16}$ ) that at $\omega \sim \omega_{e}$ the LPM effect results in $10 \%$ suppression of total (incoherent) pair creation probability. In oriented crystal $\omega_{e} \rightarrow \omega_{0}$ and at $\omega \sim \omega_{m}$ the coherent and incoherent contributions are nearly equal. So, at this photon energy one can expect $\sim 5 \%$ LPM effect for pair creation process. This perfectly agrees with performed numerical calculation. For germanium crystal $k \sim \omega_{m} / \omega_{0} \sim 1 / 5$ at $\omega \sim \omega_{m}$ (see discussion after Eq.(18)) and for this energy one can expect the LPM effect of order $\sim 1 \%$ for pair creation process (the term with $\left(\omega g / \omega_{0}\right)^{2}$ in Eq. $\left.(25)\right)$.

In just the same way the maximal influence of multiple scattering on the incoherent radiation process (see Eq.(64)) is reached in heavy elements, e.g. tungsten, at $\varepsilon \sim \varepsilon_{m} \sim \varepsilon_{0}$ where $k \sim 1$. However at $\varepsilon \sim \varepsilon_{m}$ the intensity of incoherent radiation constitutes only one tenth of coherent contribution. Owing to this the maximum of the LPM effect manifestation in the radiation process is shifted to the left up to $\varepsilon \sim \varepsilon_{t}$, where the coherent and incoherent contributions to the radiation intensity are nearly equal and $v_{0}^{2} \sim \varepsilon_{t} / \varepsilon_{0} \sim 1 / 10$. This explains essentially smaller influence of the LPM effect on radiation process.

\section{CONCLUSIONS}

So the rather prevalent assumption that the LPM effect can essentially suppress the radiation and pair creation process in oriented crystals is proved wrong due to action of axis field. On the other hand, the LPM effect can be observed in accurate measurements. For observation the LPM effect of mentioned scale in an amorphous tungsten in hard part of the spectrum of radiation process the electrons with the energy $\varepsilon \simeq 2.5 \mathrm{TeV}$ are needed (or for pair creation process the photons with energy $\omega \simeq 10 \mathrm{TeV}$ are needed). ${ }^{4}$

So in high energy region the mechanisms of radiation and pair creation by a photon are very different in an amorphous medium and in oriented crystal. In amorphous medium the radiation intensity is suppressed substantially at $\varepsilon>\varepsilon_{e}$ (or the probability of pair creation is suppressed substantially at $\omega>\omega_{e}$ ) due to the LPM effect and tends to zero at $\varepsilon \gg \varepsilon_{e}$ (or $\omega \gg$ $\left.\omega_{e}\right){ }^{4}$ In oriented crystal the coherent mechanism dominates and at $\chi_{s} \gg 1$ (or $\kappa_{s} \gg 1$ ) the radiation intensity (or probability of pair creation) is decreasing also (see Eq.(17.17) (or Eq.(12.16)) in ${ }^{8}$ ). The incoherent mechanism is suppressed and the LPM effect is suppressed more strongly as it is follow from the above discussion. It should be noted that the radiation intensity (and the probability of pair creation) in oriented crystal is always much higher than in a corresponding amorphous medium.

It's instructive to compare the LPM effect in oriented crystal for radiation and pair creation processes. The manifestation of the LPM effect is essentially different because of existence of threshold in pair creation process. The threshold energy $\omega_{m}$ is relatively high (in $\mathrm{W}$, axis $<111>, \omega_{m} \sim 8 \mathrm{GeV}$ for $\mathrm{T}=100 \mathrm{~K}$ and $\omega_{m} \sim 14 \mathrm{GeV}$ for $\mathrm{T}=293 \mathrm{~K}$ ). Below $\omega_{m}$ influence of field of axis is weak and the relative contribution of the LPM effect attains $5.5 \%$ for $\mathrm{T}=100 \mathrm{~K}{ }^{3}$ There is no threshold in radiation process and $I^{F}$ becomes larger than $I^{i n c}$ at much lower energy $\varepsilon_{t}$ and starting from this energy the influence of field of axis suppresses strongly the LPM effect. So the energy interval in which the LPM effect could appear is much narrower than for pair creation and its relative contribution is less than $1 \%$ in $\mathrm{W}$, axis $<111>$. Since value of $\varepsilon_{t}$ depends weakly on $Z$ (Eq. $(10)$ ), 
$\varepsilon_{m} \propto Z^{-1}$ (Eqs.(5), (6)) and $\varepsilon_{0} \propto Z^{-2}$ (Eq.(9)) the relative contribution of the LPM effect $\Delta$ for light elements significantly smaller. Thus, the above analysis shows that influence of multiple scattering on basic electromagnetic processes in oriented crystal (radiation and pair creation) is very limited especially for radiation process.

Let us note the important result obtained connected with decomposition of Eqs.(27) and (57) over powers of $v_{0}^{2}$. The above analysis shows that the characteristics of the processes under consideration are described quite satisfactory by the two first terms of the decomposition over $v_{0}^{2}$ (the coherent and incoherent contributions). The applicability of the third term of the decomposition $\left(\propto v_{0}^{4}\right)$ is restricted to either very low energy interval (see Eqs.(8) and (20) and corresponding comments) or very high energy region (because a weak dependence of $k$ on $\varepsilon(\omega)$ since $k \propto \varepsilon^{1 / 3}$ ). In the both limiting cases the LPM effect is negligibly small, but in the energy interval where the LPM effect could manifest itself, one has to apply the general formulas Eqs.(27) and (57).

\section{Acknowledgments}

We are grateful to U.Uggerhoj for data. The authors are indebted to the Russian Foundation for Basic Research supported in part this research by Grant 06-02-16226.

\section{References}

[1] V. N. Baier, V. M. Katkov, and V. M. Strakhovenko, "Emission of radiation by high-energy particles in oriented single crystals", Sov.Phys.JETP, 65, 686-696, 1987.

[2] V. N. Baier, V. M. Katkov, and V. M. Strakhovenko, "Production of electron-positron pairs by high-energy photons in oriented crystals",Sov.Phys.JETP, 63, 467-475, 1986.

[3] V. N. Baier, and V. M. Katkov,"Coherent and incoherent pair creation by a photon in oriented single crystals", Phys.Lett., A 346, 359-366, 2005.

[4] V. N. Baier, and V. M. Katkov, "Coherent and incoherent radiation from high-energy electron in oriented single crystals", Phys.Lett., A 353, 91-97, 2006.

[5] V. N. Baier, and V. M. Katkov,"On propagation of high-energy photon in a medium in presence of an external field" Phys.Lett., A 286, 299-308, 2001.

[6] G.Diambrini Palazzi,"'High-energy bremsstrahlung and electron pair production in thin crystals", Rev.Mod.Phys., 40, 611$631,1968$.

[7] M.Ter-Mikaelyan, High-Energy Electromagnetic Processes in Condensed Media, Wiley, NY, 1972.

[8] V. N. Baier, V. M. Katkov and V. M. Strakhovenko, Electromagnetic Processes at High Energies in Oriented Single Crystals, World Scientific Publishing Co, Singapore, 1998.

[9] V. N. Baier, V. M. Katkov, and V. M. Strakhovenko, "Incoherent radiation and pair creation in crystals", phys.stat.solidi(b), 149, 403-415, 1988.

[10] K.Kirsenom, U.Mikkelsen, E.Uggerhoj et al, "First measurement of the unique influence of spin on energy loss of ultrarelativistic electrons in strong electromagnetic fields", Phys. Rev. Lett., 87, 054801-1-4, 2001.

[11] A. Baurichter, K.Kirsenom, Yu.V.Kononetset al, "Radiation emission and its influence on the motion of multi-GeV electrons and positrons in strong crystalline fields", Phys. Rev. Lett., 79, 3415-3418, 1997.

[12] K. Kirsebom, Yu.V.Kononets, U.Mikkelsen et al, "Pair production by $5-150 \mathrm{GeV}$ photons in the strong crystalline fields of germanium, tungsten and iridium”, Nucl. Instrum. Methods, B 135, 143-148, 1998. 
[13] A. Baurichter et al, "Enhanced electromagnetic showers initiated by 20-180 GeV gamma rays on alignrd Ge crystal", Nucl. Instrum. Methods, B 152, 472-478, 1999.

[14] V. N. Baier, and V. M. Katkov, "Influence of a medium on pair photoproduction and bremsstrahlung", Phys.Rev., D 62, 036008-1-8, 2000.

[15] V. N. Baier, and V. M. Katkov, "Variation of radiation length due to LPM effect", Phys.Lett., A 327, 202-209, 2004.

[16] V. N. Baier, and V. M. Katkov, ”Concept of formation length in radiation theory", Phys.Rep., 409, 261-359, 2005. 